\title{
2d CDT is 2d Hořava-Lifshitz quantum gravity
}

\author{
Jan Ambjørn ${ }^{a, d}$, Lisa Glaser ${ }^{a}$, Yuki Sato $^{b}$ and Yoshiyuki Watabiki ${ }^{c}$
}

\author{
${ }^{a}$ The Niels Bohr Institute, Copenhagen University \\ Blegdamsvej 17, DK-2100 Copenhagen Ø, Denmark. \\ email: ambjorn@nbi.dk, glaser@nbi.dk \\ ${ }^{b}$ Department of Physics, Nagoya University, \\ Nagoya 464-8602, Japan. \\ email: ysato@th.phys.nagoya-u.ac.jp \\ ${ }^{c}$ Tokyo Institute of Technology, \\ Dept. of Physics, High Energy Theory Group, \\ 2-12-1 Oh-okayama, Meguro-ku, Tokyo 152-8551, Japan \\ email: watabiki@th.phys.titech.ac.jp \\ ${ }^{d}$ Institute for Mathematics, Astrophysics and Particle Physics \\ Radbaud University Nijmegen, \\ Heyendaalseweg 135, 6525 AJ, Nijmegen, The Netherlands
}

\begin{abstract}
Causal Dynamical Triangulations (CDT) is a lattice theory where aspects of quantum gravity can be studied. Two-dimensional CDT can be solved analytically and the continuum (quantum) Hamiltonian obtained. In this article we show that this continuum Hamiltonian is the one obtained by quantizing twodimensional projectable Hořava-Lifshitz gravity.
\end{abstract}




\section{Introduction}

Causal Dynamical Triangulations (CDT) provides a non-perturbative lattice regularization for a quantum theory of geometries which are characterized by allowing a time foliation (for a recent review see [1]). While originally formulated for spacetimes with Lorentzian signature, the formulation is such that one can rotate to Euclidean signature and perform the path integral there. In this sector one can prove that the lattice theory has a transfer matrix which is reflection positive. One thus expects that a continuum theory obtained from the lattice theory will have a unitary time-evolution.

The features just mentioned are by large the same features as Peter Hořava imposed on what is now known as Hořava-Lifshitz gravity [2]. Thus CDT should provide a lattice regularization of Hořava-Lifshitz gravity (HL). This was first suggested in 3] where it was noted that the spectral dimension in HL gravity agreed with the one measured in CDT [4. Indeed, there is good evidence that CDT can provide a regularization for HL gravity [5]. So far this evidence is based on numerical simulations in three and four dimensions? Unfortunately it is difficult to solve the lattice theory analytically and equally difficult to address details of the continuum renormalizable quantum theories in these dimensions. However, the two-dimensional theory provides a simple test: the lattice theory can be solved analytically ([6]) and in $2 \mathrm{~d}$ we can analyze the corresponding continuum HL quantum theory and show that it agrees with 2d CDT.

\section{Projectable Hořava-Lifshitz gravity}

In HL it is assumed that spacetime has a time foliation and that the theory is invariant under spatial diffeomorphisms as well as time redefinitions, i.e. so-called foliation preserving diffeomorphisms:

$$
t \rightarrow t+\xi^{0}(t), \quad x^{i} \rightarrow x^{i}+\xi^{i}(t, x),
$$

where $i=1, \ldots, d, d$ being the dimension of space. We will be interested in the very simplest version of this theory which contains at most second order derivatives of the metric. It is convenient to use the ADM-decomposition of the metric

$$
g_{\mu \nu}(x, t) d x^{\mu} d x^{\nu}=-N(x, t)^{2} d t^{2}+g_{i j}(x, t)\left(d x^{i}+N^{i}(x, t) d t\right)\left(d x^{j}+N^{j}(x, t) d t\right),
$$

\footnotetext{
${ }^{1}$ Of course there is also in addition the possibility that one obtains a quantum theory of standard general relativity (GR) for special values of the bare lattice coupling constants, and the search for such "isotropic" points is an important part of the CDT program.
} 
where $N(x, t)$ is the lapse function and $N^{i}(x, t)$ is the shift vector. The action is given by

$$
I=\frac{1}{\kappa} \int d t d^{d} x \sqrt{g} N\left[\left(K_{i j} K^{i j}-\lambda K^{2}\right)+R-2 \Lambda\right] .
$$

Here $g_{i j}$ is the spatial metric on the foliation, $R$ the corresponding scalar curvature, and $K_{i j}$ is the extrinsic curvature defined by the lapse function $N$ and the shift vector $N^{i}$ as

$$
K_{i j}=\frac{1}{2 N}\left(\partial_{0} g_{i j}-\nabla_{i} N_{j}-\nabla_{j} N_{i}\right)
$$

where $\nabla_{i}$ is the covariant derivative associated with $g_{i j}$, and $K=g^{i j} K_{i j}$. Finally $\kappa$ is the gravitational coupling constant analogous to the Newton constant in GR, $\Lambda$ the cosmological constant and $\lambda$ a dimensionless parameter. If $\lambda=1$ one recovers GR and in this case $I$ is just the integrated intrinsic scalar curvature, i.e. the standard GR action. However, for $\lambda \neq 1 I$ is only invariant under the foliation preserving coordinate transformations (1).

As is standard in the ADM decomposition one can (up to boundary terms) rewrite the action as

$$
I=\int d t \int d^{d} x\left[\pi^{i j} \partial_{0} g_{i j}-N \mathcal{H}-N_{i} \mathcal{H}^{i}\right]
$$

where

$$
\mathcal{H}=\frac{\kappa}{\sqrt{g}}\left(\pi^{i j} \pi_{i j}-\frac{\lambda}{d \lambda-1} \pi^{2}\right)-\frac{\sqrt{g}}{\kappa}(R-2 \Lambda), \quad \mathcal{H}^{i}=-2 \nabla_{j} \pi^{i j}
$$

and where the canonical momenta are

$$
\pi^{i j}=\frac{\partial \mathcal{L}}{\partial\left(\partial_{0} g_{i j}\right)}=\frac{\sqrt{g}}{\kappa}\left(K^{i j}-\lambda g^{i j} K\right), \quad \pi=g_{i j} \pi^{i j} .
$$

Here $\mathcal{L}$ is the Lagrangian density corresponding to the action $I . \pi^{i j}$ is a tensor density and $\nabla_{j} \pi^{i j}$ is the standard covariant derivative of a tensor density. $\mathcal{H}$ is the Hamiltonian constraint and $\mathcal{H}^{i}$ the momentum constraints and the lapse and shift functions appear only as Lagrange multipliers. The functional form of eq. (5) is identical to the standard Hamiltonian form of the gravitational action in GR and the Hamiltonian

$$
H=\int d^{d} x\left[N \mathcal{H}+N_{i} \mathcal{H}^{i}\right]
$$

is proportional to the constraints like in GR.

In the following we will consider only $1+1$ dimensions. In addition we restrict ourselves to so-called projectable HL gravity, where one assumes that the 
lapse function $N(x, t)$ depends only on $t$. This restriction is compatible with the foliation preserving diffeomorphisms. In $1+1$ dimensions $\kappa$ is a dimensionless coupling constant and it will play no role. We thus set $\kappa=1$ in the following. The $1+1$ dimensional action simplifies dramatically. We have $R=0$ since $d=1$ and further $g_{11}=g$. It is convenient to use as canonical variable $\gamma=\sqrt{g}$ :

$$
I=\int d t d x N \gamma\left[(1-\lambda) K^{2}-2 \Lambda\right]
$$

where

$$
K=\frac{1}{N}\left(\frac{1}{\gamma} \partial_{0} \gamma-\frac{1}{\gamma^{2}} \partial_{1} N_{1}+\frac{N_{1}}{\gamma^{3}} \partial_{1} \gamma\right)
$$

The conjugate momentum of our metric variable $\gamma$ is

$$
\pi^{\gamma}=\frac{\partial \mathcal{L}}{\partial\left(\partial_{0} \gamma\right)}=2(1-\lambda) K, \quad\left\{\gamma(x, t), \pi^{\gamma}(y, t)\right\}=\delta(x-y),
$$

where the last equation states the Poisson bracket between $\gamma$ and $\pi^{\gamma}$. Eq. (8) now reads:

$$
H=\int d x\left[N \mathcal{H}+N_{1} \mathcal{H}^{1}\right], \quad \mathcal{H}=\gamma \frac{\left(\pi^{\gamma}\right)^{2}}{4(1-\lambda)}+2 \Lambda \gamma, \quad \mathcal{H}^{1}=-\frac{\partial_{1} \pi^{\gamma}}{\gamma} .
$$

A great simplification comes from the momentum constraint

$$
\mathcal{H}^{1}=0 \quad \text { i.e. } \quad \pi^{\gamma}(x, t)=\pi^{\gamma}(t)
$$

On this constraint surface $H$ can be expressed as follows:

$$
H=N(t)\left(L(t) \frac{\left(\pi^{\gamma}(t)\right)^{2}}{4(1-\lambda)}+2 \Lambda L(t)\right), \quad L(t):=\int d x \gamma(x, t),
$$

entirely in terms of variables with no $x$ dependence. Note that both $L(t)$ and $\pi^{\gamma}(t)$ are invariant under the foliation preserving diffeomorphisms (1). $N(t)$ can be regarded as the Lagrange multiplier of the Hamiltonian constraint. This fixes $\pi^{\gamma}(t)$ to be a $t$ independent constant provided $(\lambda-1) \Lambda>0$ :

$$
\left(\pi^{\gamma}\right)^{2}=8(\lambda-1) \Lambda
$$

This equation tells us that the constraint surface is a surface of constant extrinsic curvature, provided the rhs is positive. It clearly depends on $\lambda$ and $\Lambda$ whether or not that is the case. If the rhs of eq. (15) is negative the only solution to the constraint coming from $N(t)$ is $L(t)=0$. In this case, if we want to start with 
a dynamical system with a genuine non-trivial classical solution, we can add the constraint that the volume of spacetime should be a constant $A$. Effectively this means that the cosmological constant is treated as a Lagrange multiplier, which after solving the eom is fixed such that the corresponding spacetime volume is $A 2^{2}$. However, using the path integral quantization, as we will in the next section, there is no formal problem associated with a quantization around $L(t)=0$. With this understanding the classical dynamics of $L(t)$ is entirely determined by the action

$$
S=\int d t\left(\frac{\dot{L}^{2}}{4 N(t) L(t)}-\tilde{\Lambda} N(t) L(t)\right), \quad \tilde{\Lambda}=\frac{\Lambda}{2(1-\lambda)},
$$

and the corresponding Hamiltonian is

$$
H=N(t)\left(L \Pi^{2}+\tilde{\Lambda} L\right), \quad\{L(t), \Pi(t)\}=1 .
$$

\section{Quantization}

In order to make contact to CDT we now perform a formal rotation to Euclidean signature and use the path integral to quantize the theory. Thanks to the existence of a time foliation in HL gravity we can think of this as a formal substitution $t \rightarrow i t$ which changes the sign of the first term in 11$]^{3}$. Note that in projectable HL gravity proper time

$$
t_{p}(t):=\int_{0}^{t} d t^{\prime} N\left(t^{\prime}\right)
$$

is a physical observable: it cannot be changed by an allowed redefinition (1) of spacetime. Since $L(t)$ is also invariant under time redefinition we can, in HL gravity, view $L\left(t_{p}\right)$ as an observable developing in "real" observable time $t_{p}$. In the quantum theory it is thus a perfectly legitimate question to ask for the probability amplitude that the universe evolves from a state of length $L_{1}$ (i.e. an eigenstate of the operator $\hat{L}$ with eigenvalue $\left.L_{1}\right)$ to a state of length $L_{2}$ in proper time $T$. This amplitude $G\left(L_{2}, L_{1} ; T\right)$ is precisely given by the path integral (we give the

\footnotetext{
${ }^{2}$ The same problem is encountered in $2 \mathrm{~d}$ Liouville gravity, and the same solution is used: the Liouville equation can be written as $R=-\Lambda$, where $R$ is the $2 \mathrm{~d}$ scalar curvature. But since $\int \sqrt{g} R=2 \pi \chi, \chi$ being the Euler characteristics of the $2 \mathrm{~d}$ manifold, there is no classical solution for positive $\Lambda$ and $\chi$ not negative unless one e.g. fixes the total spacetime volume.

${ }^{3}$ Of course the metric functions appearing in (1) have in general no such well defined analytic continuation. The same is true for fields $\phi(t, x)$ when one performs the rotation to Euclidean signature: general fields appearing in the path integral do not allow such a rotation. In fact they are with probability one not even analytic functions of $t$. However, certain amplitudes, calculated in flat Lorentzian spacetime can be rotated to amplitudes calculated in Euclidean spacetime.
} 
Euclidean version)

$$
G\left(L_{2}, L_{1} ; T\right)=\int \frac{\mathcal{D} N(t)}{\operatorname{Diff}[0,1]} \int \mathcal{D} L(t) \mathrm{e}^{-S_{E}[N(t), L(t)]}
$$

Here $S_{E}$ is the action (16) rotated to Euclidean signature

$$
S_{E}=\int d t\left(\frac{\dot{L}^{2}}{4 N(t) L(t)}+\tilde{\Lambda} N(t) L(t)\right)
$$

and we integrate over all functions

$$
\begin{gathered}
N: t \rightarrow N(t), \quad t \in[0,1], \quad \int_{0}^{1} d t N(t)=T, \\
L: t \rightarrow L(t), \quad t \in[0,1], \quad L(0)=L_{1}, \quad L(1)=L_{2} .
\end{gathered}
$$

By standard gauge fixing to proper time $N(t)$ drops out of the action and any reference to $N(t)$ disappears in $(19)$. We are thus left with an ordinary path integral where the time is proper time, which, as discussed above, is an observable in HL gravity. $G\left(L_{2}, L_{1} ; T\right)$ defines the quantum Hamiltonian $\hat{H}$ by:

$$
\begin{aligned}
G\left(L_{2}, L_{1} ; T\right) & =\left\langle L_{2}\left|\mathrm{e}^{-T \hat{H}}\right| L_{1}\right\rangle, \\
G\left(L_{2}, L_{1} ; \varepsilon\right) & =\left\langle L_{2}\left|\left(\hat{1}-\varepsilon \hat{H}+O\left(\varepsilon^{3 / 2}\right)\right)\right| L_{1}\right\rangle .
\end{aligned}
$$

In order to extract $\hat{H}$ from 23 one uses the basic definition of the path integral. The proper time interval $[0, T]$ is subdivided in pieces of length $\varepsilon$, (assuming $\left.\mathcal{D} L(t)=\prod d L\left(t_{i}\right)\right)$

$$
\int_{0}^{\infty} d L_{1} G\left(L_{2}, L_{1} ; \varepsilon\right) \psi\left(L_{1}\right)=\psi\left(L_{2}\right)-\varepsilon(\hat{H} \psi)\left(L_{2}\right)+O\left(\varepsilon^{3 / 2}\right) .
$$

The result of this standard calculation is:

$$
\hat{H}=-\frac{d}{d L} L \frac{d}{d L}+\tilde{\Lambda} L .
$$

This is precisely the CDT Hamiltonian derived in [7] and [8].

A much simpler way to derive the Hamiltonian is to gauge fix (17) by choosing proper time. Then we have classically $H=L \Pi^{2}+\tilde{\Lambda} L$, and

$$
\{L, \Pi\}=1 \rightarrow[\hat{L}, \hat{\Pi}]=i \quad \text { by } \quad \hat{\Pi}=-i \frac{d}{d L} .
$$


However, this procedure does not resolve the operator ordering ambiguity that arises when $L \Pi^{2}$ is promoted to a quantum operator. In fact we have three options

$$
\left(L \Pi^{2}\right)_{0}=-\frac{d}{d L} L \frac{d}{d L}, \quad\left(L \Pi^{2}\right)_{-1}=-L \frac{d^{2}}{d L^{2}}, \quad\left(L \Pi^{2}\right)_{1}=-\frac{d^{2}}{d L^{2}} L
$$

Option 0 leads to an operator which is Hermitian when we use the flat measure $d L$ on the positive real axis (and appropriate boundary conditions). Option -1 leads to a Hermitian operator if we choose the measure $d L / L$, and similarly option 1 leads to a Hermitian operator when we use $L d L$ (all with appropriate boundary conditions, of course). For these different choices of Hamiltonians, $\hat{H}_{i}$, $i=-1,0,1$, the amplitudes $G_{i}\left(L_{2}, L_{1} ; T\right)$ satisfy different composition rules:

$$
G_{i}\left(L_{2}, L_{1} ; T_{1}+T_{2}\right)=\int_{0}^{\infty} d L G\left(L_{2}, L ; T_{2}\right) L^{i} G\left(L, L_{1} ; T_{1}\right)
$$

We can of course derive the Hamiltonians $\hat{H}_{i}, i= \pm 1$ from the continuum path integral in the same way as we derived (26), provided we start out with the measures

$$
\mathcal{D} L(t)=\lim _{\varepsilon \rightarrow 0} \prod_{k=1}^{T / \varepsilon} L^{i}\left(t_{k}\right) d L\left(t_{k}\right) .
$$

The geometrical interpretation of these measures for $i= \pm 1$ was already given in the original CDT article [6]. The measure $L d L$ corresponds to considering spatial universes which are closed (i.e. a circle) and where no points are marked on the boundaries. These universes come with symmetry factors $1 / L$. However, when these universes are glued together, as in (29), the boundaries become internal. To then correctly count the different surfaces that arise from gluing one needs to compensate for the $1 / L$ factor by multiplying with $L$. Similarly, the measure $d L / L$ arises from gluing together cylinder amplitudes where one has chosen to mark a point on the boundary. Finally, as shown in [7] the flat measure $d L$ describes the situation where we have open boundaries of length $L$. In this case there is no symmetry factor associated with the gluing. It is remarkable that all operator orderings have a simple geometric realization.

In our projectable HL gravity the classical Hamiltonian constraint $L \Pi^{2}+\tilde{\Lambda} L=$ 0 on the constraint surface leads to the quantum statement that the HartleHawking wave function for a closed universe satisfies

$$
\hat{H}_{ \pm 1} W^{( \pm 1)}(L)=0, \quad \hat{H}=L \hat{\Pi}^{2}{ }_{ \pm 1}+\tilde{\Lambda} \hat{L}
$$

depending on how we treat the boundary conditions. Eq. (31) is nothing but the Wheeler-DeWitt equation. The Hartle-Hawking ground state of the universe 
describes the "propagation" in Euclidean spacetime from "nothing" to a given space of length $L$. This is what in the context of CDT has been called the "disk" function since the topology of spacetime is that of a disk if we start from "nothing". Explicitly the disk functions are [6]

$$
\begin{gathered}
W^{( \pm 1)}(L)=\int_{0}^{\infty} d T G_{ \pm 1}\left(L, L_{1}=0 ; T\right) ; \\
W^{(1)}(L)=\mathrm{e}^{-\sqrt{\tilde{\Lambda}} L} / L, \quad W^{(-1)}(L)=\mathrm{e}^{-\sqrt{\tilde{\Lambda}} L} .
\end{gathered}
$$

Although the disk functions $W^{( \pm 1)}(L)$ satisfy $\hat{H}_{ \pm 1} W^{( \pm 1)}(L)=0$ they are not normalizable wavefunctions with respect to the measures $d L L^{ \pm 1}$, respectively (nothing tells us that the wavefunction of the universe has to be normalizable!). The energy spectra of $\hat{H}_{ \pm 1}$ are positive definite (for $\tilde{\Lambda}>0$ ) with eigenvalues $E_{ \pm 1}(n)=2 \sqrt{\tilde{\Lambda}}(n+1), n=0,1, \ldots$ (see [9] where also the Hartle-Hawking wave function which includes the summation over spacetime topologies can be found).

\section{Discussion}

We have identified the continuum theory corresponding to $2 \mathrm{~d}$ CDT. It is projectable 2d HL gravity with $\lambda<1$ and $\Lambda>0$. By expressing the HL theory in terms of proper time $T$ and the scale factor of the universe $L(T)$, and then quantizing the theory, we obtained a quantum theory identical to the continuum limit of CDT.

2d HL gravity offers us an interesting laboratory for addressing the standard conundrum of rotating to the Euclidean sector: if we choose $\lambda>1$ we imitate the standard situation we believe is realized in our universe for positive $\Lambda$ : it is expanding (or contracting) exponentially in (proper) time $T$. However, the "kinetic" scale factor term which leads to this expansion has the wrong sign compared to an ordinary kinetic term (precisely as in ordinary GR), causing disaster when naively rotated to the Euclidean sector. If we choose $\lambda<1$ the situation is healthy compared to an ordinary dynamical system, but it does not correspond to a dynamical system resembling our universe unless we choose $\Lambda<0$. At a classical level there is no difference since we can just change the sign of $S$ as we have done by unifying the situations in (16). However, when coupling to matter and in higher dimensions where one might also have transverse physical field degrees of freedom one might not have that option. This needs to be analyzed.

It might be interesting to analyze non-projectable $2 \mathrm{~d}$ HL gravity. In this case one has the "many" fingered proper time of Wheeler:

$$
t_{p}(t, x)=\int_{0}^{t} d t^{\prime} N\left(t^{\prime}, x\right),
$$


which allows us to consider more general amplitudes. It is tempting to conjecture that the quantum theory corresponding to non-projectable $2 \mathrm{~d}$ HL is so-called generalized CDT [10], which also allows more general amplitudes. Generalized CDT was originally formulated in the continuum and has a continuum string field theory representation [11] in the same way as "ordinary" dynamical triangulation (DT) has [12. Later a discretized representation of generalized CDT was found and it is universal in the same way as DT (and has a matrix model representation in the same way as DT [13]). More specifically any reasonable class of so-called planar maps has as its continuum limit Euclidean Liouville quantum gravity. It has recently been shown that (continuum) generalized CDT is described as the continuum limit of any reasonable class of planar maps with a finite number of faces [14. So we understand in quite some detail both the regularized theory and its continuum limit, and what is lacking is a continuum classical theory which when quantized is generalized CDT. Non-projectable 2d Hořava-Lifshitz gravity is a candidate.

Finally, to avoid confusion, let us comment on the relation between $2 \mathrm{~d}$ HL gravity and $2 \mathrm{~d}$ GR. Since the Einstein action is topological in $2 \mathrm{~d}$ we have to define what we mean by $2 d$ GR. Here we will think about $2 d$ GR as the quantum theory defined by Liouville gravity, i.e. in the Euclidean context the theory which results after integrating over all geometry of a fixed topology. It can still depend on diffeomorphism invariant boundary data, i.e. the length of the boundaries. However, in this theory there is no propagating degrees of freedom (the Liouville field is not a gravitational field degree of freedom) and the only scale is set by the cosmological constant. The same is true for CDT or quantized 2d HL gravity. Since we are only considering theories with quadratic derivative terms (both theories are renormalizable without adding higher derivative terms) there is no "flow" of $2 \mathrm{~d}$ HL to an isotropic point, as is the case in higher dimensions. It does not mean that the two theories are not related. In fact there exists an explicit map between the theories [15] and one obtains CDT by integrating out so-called baby universes present in DT. Generalized CDT presents in some sense an interpolation between CDT and DT and again it is tempting to suggest that non-projectable quantum HL can provide us with such an interpolation with the parameter $\lambda \rightarrow 1$ being the limit where Liouville quantum gravity should be recovered.

Acknowledgments. LG would like to thank Luís Pires for helpful discussions. JA and LG were partly supported by the Danish Research Council via the grant "Quantum gravity and the role of Black holes". JA and YW were partly supported by the the EU through the ERC Advanced Grant 291092, "Exploring the Quantum Universe" (EQU). YS and YW thank the Niels Bohr Institute for warm hospitality. YS is supported by the Grant-in-Aid for Nagoya University 
Global COE Program, "Quest for Fundamental Principles in the Universe: from Particles to the Solar System and the Cosmos".

\section{References}

[1] J. Ambjorn, A. Goerlich, J. Jurkiewicz and R. Loll, Physics Reports 519 (2012) 127 [arXiv:1203.3591 [hep-th]].

[2] P. Horava, Phys. Rev. D 79 (2009) 084008 arXiv:0901.3775 [hep-th]].

[3] P. Horava, Phys. Rev. Lett. 102 (2009) 161301 arXiv:0902.3657 [hep-th]].

[4] J. Ambjorn, J. Jurkiewicz and R. Loll, Phys. Lett. B 607 (2005) 205 hep-th/0411152.

[5] J. Ambjorn, A. Gorlich, S. Jordan, J. Jurkiewicz and R. Loll, Phys. Lett. B 690 (2010) 413 arXiv:1002.3298 [hep-th]].

C. Anderson, S. J. Carlip, J. H. Cooperman, P. Horava, R. K. Kommu and P. R. Zulkowski, Phys. Rev. D 85 (2012) 044027 arXiv:1111.6634 [hep-th]]. J. Ambjorn, S. Jordan, J. Jurkiewicz and R. Loll, Phys. Rev. Lett. 107 (2011) 211303 arXiv:1108.3932 [hep-th]].

[6] J. Ambjorn and R. Loll, Nucl. Phys. B 536 (1998) 407 hep-th/9805108].

[7] P. Di Francesco, E. Guitter and C. Kristjansen, Nucl. Phys. B 567 (2000) 515 hep-th/9907084.

[8] B. Durhuus and C. W. H. Lee, Nucl. Phys. B 623 (2002) 201 hep-th/0108149].

[9] J. Ambjorn, R. Loll, W. Westra and S. Zohren, Phys. Lett. B 678 (2009) 227 arXiv:0905.2108 [hep-th]]. Phys. Lett. B 680 (2009) 359 arXiv:0908.4224 [hep-th]].

S. Zohren, arXiv:0905.0213 [hep-th].

[10] J. Ambjorn, R. Loll, W. Westra and S. Zohren, JHEP 0712 (2007) 017 arXiv:0709.2784 [gr-qc]]. 
[11] J. Ambjorn, R. Loll, Y. Watabiki, W. Westra and S. Zohren, JHEP 0805 (2008) 032 arXiv:0802.0719 [hep-th]].

[12] N. Ishibashi and H. Kawai, Phys. Lett. B 314 (1993) 190 hep-th/9307045].

Phys. Lett. B 322 (1994) 67 |hep-th/9312047|.

[13] J. Ambjorn, R. Loll, Y. Watabiki, W. Westra and S. Zohren, Phys. Lett. B 665 (2008) 252 arXiv:0804.0252 [hep-th]]. Phys. Lett. B 670 (2008) 224 arXiv:0810.2408 [hep-th]].

[14] J. Ambjorn and T. G. Budd, arXiv:1302.1763 [hep-th].

[15] J. Ambjorn, J. Correia, C. Kristjansen and R. Loll, Phys. Lett. B 475 (2000) 24 [hep-th/9912267]. 